\title{
Induction of Myogenic Differentiation Improves Chemosensitivity of Chemoresistant Cells in Soft-Tissue Sarcoma Cell Lines
}

\author{
Lucy E. Dawson, ${ }^{1}$ Luca D'Agostino, ${ }^{1}$ Abraham A. Hakim, ${ }^{2}$ Richard D. Lackman, ${ }^{1,3}$ \\ Spencer A. Brown, ${ }^{1}$ Richard B. Sensenig, ${ }^{1}$ Zeus A. Antonello $\mathbb{D}^{1,2}$ and Igor I. Kuzin ${ }^{1,2}$ \\ ${ }^{1}$ Cooper University Hospital, Camden, NJ, USA \\ ${ }^{2}$ Cooper Medical School of Rowan University, Camden, NJ, USA \\ ${ }^{3} \mathrm{MD}$ Anderson Cancer Center at Cooper, Camden, NJ, USA
}

Correspondence should be addressed to Zeus A. Antonello; antonello-zeus@cooperhealth.edu and Igor I. Kuzin; kuzin-igor@cooperhealth.edu

Received 22 October 2019; Revised 30 January 2020; Accepted 7 February 2020; Published 26 March 2020

Academic Editor: Andreas Leithner

Copyright (c) 2020 Lucy E. Dawson et al. This is an open access article distributed under the Creative Commons Attribution License, which permits unrestricted use, distribution, and reproduction in any medium, provided the original work is properly cited.

\begin{abstract}
Rhabdomyosarcoma (RMS) and rhabdoid tumors (RT) are rare soft-tissue malignancies with the highest incidence in infants, children, and adolescents. Advanced, recurrent, and/or metastatic RMS and RT exhibit poor response to treatment. One of the main mechanisms behind resistance to treatment is believed to be intratumoral heterogeneity. In this study, we investigated the myogenic determination factor 1 (MYOD1) and Noggin (NOG) markers in an embryonal RMS (ERMS) cell line and an RT cell line and the differential response of the MYOD1 and NOG expressing subpopulations to chemotherapy. Importantly, we found that these markers together identify a subpopulation of cells (MYOD1+ NOG+ cells) with primary resistance to Vincristine and Doxorubicin, two commonly used chemotherapies for ERMS and RT. The chemoresistant MYOD1+ NOG+ cells express markers of undifferentiated cells such as myogenin and ID1. Combination of Vincristine with TPA/GSK126, a drug combination shown to induce differentiation of RMS cell lines, is able to partially overcome MYOD1/NOG cells chemoresistance.
\end{abstract}

\section{Introduction}

Rhabdomyosarcoma (RMS) and rhabdoid tumors (RT) are rare soft-tissue malignancies with the highest incidence in infants, children, and adolescents. About 400 to 500 new cases of RMS and only about 15 new cases of RT occur each year in the United States, comprising approximately $3 \%$ of all childhood cancers. Although RMS rarely occurs in adults, the outcomes are significantly worse [1]. Many adult patients with advanced RMS die because their cancer exhibits or develops resistance to available therapies. RMS is comprised of two main histological variants, alveolar and embryonal (ERMS). ERMS has a more complex and heterogeneous genetic profile but has an overall better outcome, as high as $90 \%$ 5-year survival for the low-risk group [2]. However, when ERMSs are advanced, recurrent, and/or metastatic, they are classified as high risk and exhibit poor response to treatment (chemoresistance), having a progression-free survival less than 1.5 years with a 5 -year survival rate as low as $20 \%$ [3-5]. In both children and adults, RMS and RT are treated with a combination of therapies including surgery, radiation, and chemotherapy $[6,7]$.

One of the main mechanisms behind resistance to treatment and recurrence is believed to be intratumoral heterogeneity. Heterogeneity in genomic, transcriptomic, and proteomic profiles among the cells constituting the tumor manifests as a differential response to the applied therapies [8-10]. Although clinical tumors may respond by regressing in size or even becoming undetectable upon treatment, therapeutic intervention may facilitate the 
expansion of an initially small population of nonresponsive cells and reconstitute the primary tumor and/or metastasize [11]. Intratumoral heterogeneity represents therefore a major obstacle to effective cancer treatment [12]. Both main variants of RMS and RT have been reported to have intratumoral heterogeneity in patients $[13,14]$. In embryonal rhabdomyosarcoma, intratumor diversity has been correlated with reduced survival [15] and it has been shown to change under treatment in patients $[16,17]$.

In order to devise therapeutic approaches able to target a heterogeneous tumor population, it is therefore important not only to characterize the different tumor subpopulations but also to understand how cell subpopulations may change upon treatment. Such information can guide the design of high-order combined therapies [11]; however, only limited data exist regarding RMS and RT intratumor heterogeneity changes under treatment.

In this study, the differential response to chemotherapy associated with the heterogeneity of myogenic determination factor 1 (MYOD1) and Noggin (NOG) markers in ERMS and RT cell lines was investigated. The RD cell line, one of the most commonly used for RMS investigations [18], was examined as well as the A-204 cell line, originally identified as RMS but later classified as a rhabdoid tumor (RT) [19]. RMS tumors have been reported to be positive for MYOD1 with marked heterogeneity between cells [18], while RT are believed to be negative for MYOD1 [20, 21]. MYOD1 is one of the four myogenic regulatory genes that drive differentiation of muscle cell precursors to mature muscle cells, and it has been shown to be sufficient to convert nonmuscle cells into myoblast-like cells [22]. Myogenic transcription factors such as MYOD1 are normally tightly regulated during homeostasis and tissue repair [22, 23], but in RMS, MYOD1 is deregulated or mutated, resulting in reduced survival of the patients $[15,24]$. NOG is another tightly regulated protein required for correct muscle morphogenesis [25] and adult muscle homeostasis [26] and repair [27]. NOG antagonizes bone morphogenetic proteins (BMPs) which, by binding to BMP-receptors, modulate proliferation and differentiation. Inhibitors of differentiation (Id) proteins are important downstream effectors of BMP signaling and are deregulated in several cancers [28]. In myoblasts, Id proteins inhibit cell differentiation and potentiate cell proliferation by sequestering and antagonizing MYOD1 and myogenin transcription activity [29]. In RMS, morphogenetic signaling is aberrant leading to altered proliferation and differentiation of myogenic precursors and/or differentiated cells [30]. Further analysis of the myogenic determinant MYOD1 and the morphogenetic protein NOG could provide insights into the mechanisms of chemoresistance, possibly leading to improved treatments and clinical prognoses.

\section{Materials and Methods}

2.1. Cell Lines. RD (ATCC ${ }^{\circledR}$ CCL-136 ${ }^{\mathrm{TM}}$ ) and A-204 (ATCC ${ }^{\circledR} \mathrm{HTB}^{\mathrm{T}} 82^{\mathrm{TM}}$ ) were purchased from American Type Culture Collection (Manassas, VA) and cultured under standard conditions $\left(37^{\circ} \mathrm{C}, 5 \% \mathrm{CO}_{2}\right)$ in DMEM High
Glucose supplemented with $10 \%$ fetal bovine serum. Cells were seeded 24 hrs prior to each assay as indicated below.

2.2. Drugs Preparation and Treatments. All drugs were prepared and stored as per manufacturer indications. In detail, Doxorubicin (Sigma Aldrich, St. Louis, MO), 12-Otetradecanoylphorbol-13-acetate (TPA) (EMD Millipore, Temecula, CA), and GSK126 (AdooQ Bioscience, Irvine, CA) were resuspended in $100 \%$ DMSO and stored at $-20^{\circ} \mathrm{C}$. Vincristine (AdipoGen, San Diego, CA) was resuspended in sterile water and stored at $4^{\circ} \mathrm{C}$. Cells were treated after 24 hours from seeding in 6-well plates at the doses indicated in each figure. Drugs were diluted in full media and final DMSO concentration on cells never exceeded $0.01 \%$.

2.3. Flow Cytometry. Intracellular staining was performed on fixed and permeabilized RMS and RT cell suspensions with Transcription Factor Buffer Set (BD Biosciences, Jose, CA) prior to staining with fluorochrome-conjugated antihuman antibodies following manufacturer's protocol with minor modifications. Briefly, cells were incubated in Fix/ Perm buffer for $40 \mathrm{~min}$, washed with Perm/Wash buffer, and incubated for $15 \mathrm{~min}$ with $2 \% \mathrm{rabbit} / 2 \%$ mouse normal sera to block nonspecific binding. Cells were then stained for $2 \mathrm{hrs}$ with mixture of fluorochrome-conjugated antibodies in Perm/Wash buffer: Bcl-2 (clone 100) from BioLegend, MYOD1 (polyclonal) and NOG (polyclonal) from Bioss Antibodies, MYOD1 (clone SPM427) and myogenin (clone MGN185 + F5D) from Novus Biologicals, and ID1 (clone B-8) from Santa Cruz Biotechnology, followed by staining with PE-streptavidin (Invitrogen) if biotinylated antibody was included in the staining mixture. The optimal antibody dilution was established by titration curve. Matching isotype controls were prepared as per standard flow cytometry protocols to determine background signals. All steps were performed on ice and samples kept in the dark. Flow cytometry was performed using an 8-color Stratedigm (San Jose, CA) S1000EX apparatus. Data were analyzed using CellCapTure software (Stratedigm).

2.4. Cell Proliferation Assay. Cell proliferation assays were conducted using CellTrace ${ }^{\mathrm{TM}}$ Violet cell proliferation kit according to the manufacturer's protocol (Invitrogen, Carlsbad, CA). Briefly, cells were incubated in $5 \mu \mathrm{M}$ of dye in PBS solution for $20 \mathrm{~min}$ at room temperature, washed with culture medium, seeded into 6 -well plates at $0.4 \times 10^{6}$ cells per well, and cultured for 4 days. Plates were then washed with PBS to remove nonadherent dead cells, and live adherent cells were harvested with $0.25 \%$ Trypsin/10 mM EDTA in PBS, stained for intracellular markers, and analyzed by flow cytometry.

2.5. Cell Chemoresistance Assay. Cells were seeded into 6well plate at $0.4 \times 10^{6}$ cells per well, $24 \mathrm{hrs}$ prior to treatment with Vincristine (1 and $10 \mathrm{nM}$ ) or Doxorubicin (0.1 and $1 \mu \mathrm{M})$ for $48 \mathrm{hrs}$. For the analysis, plates were washed with PBS to remove dead cells, adherent cells were harvested with 
Trypsin/EDTA, and viability was determined with Automated Cell Counter NC-200 (Chemometec). After viability evaluation, cells were stained for flow cytometry analysis.

2.6. Induction of Myogenic Differentiation. Cells were seeded into 6-well plate at $0.4 \times 10^{6}$ cells per well and after $24 \mathrm{hrs}$ cultured in the presence of 12-O-tetradecanoylphorbol-13acetate (TPA) (EMD Millipore, Temecula, CA) and GSK126 (AdooQ Bioscience, Irvine, CA), at 0.1 and $5 \mu \mathrm{M}$, respectively, as previously described [31]. Cells were harvested at the indicated time points, counted, and stained for intracellular markers.

2.7. Statistical Analysis. All statistical analyses were performed using GraphPad Prism 6 (La Jolla, CA) software. One-way ANOVA test with Tukey's correction for multiple comparisons was used to assess the differences among subpopulations in treated and nontreated cell cultures, with $\alpha$ set to 0.05 . D'Agostino \& Pearson normality test had been used beforehand to validate the data normality distribution. In some instances, a paired two-tailed $t$-test has been used.

\section{Results}

3.1. Heterogeneity of MYOD1 and Noggin Expression. The protein expression of MYOD1 and Noggin genes in RD and A-204 cell lines were evaluated by flow cytometry to assess the distribution of these markers among the cell population. RMS tumors have been reported to be positive for MYOD1 with marked heterogeneity between cells [18], while RT are believed to be negative for MYOD1 [20, 21]. Interestingly, we found MYOD1 to be expressed in approximately 5 to $25 \%$ of RD cells and 1 to $10 \%$ of A-204 cells, while the majority of cells $(\geq 80 \%)$ had MYOD1 below detection levels (Table 1). NOG positive cells (NOG+) always constitute the majority of cells in both RD and A-204 (30 to 90\%). Of this population, $10-25 \%$ and $1-10 \%$ are also positive for MYOD1 (MYOD1+ NOG+) in RD and A-204, respectively. The majority ( $>90 \%)$ of MYOD1+ cells were also positive for NOG while single positive cells (MYOD1+ NOG-) were less than $5 \%$ of the MYOD1 population and less than $1 \%$ of the total, for both RD and A-204. Importantly, the variability among the observed percentages is high and the populations identified by these 2 markers are in continuum (Figure 1(a)), which strongly suggests dynamic regulation of the expression of the markers and transition of expression profiles among the subpopulations. Further experiments were performed to determine the dynamic of these markers upon chemotherapy and to assess if any of these cell subpopulations may be associated with increased chemoresistance or chemosensitivity.

3.2. MYOD1/NOG Expressing Cells Show Higher Levels of Chemoresistance. RD and A-204 cells were treated with Vincristine and Doxorubicin, two commonly used chemotherapeutic agents for RMS [32, 33], to examine expression profiles of MYOD1 and NOG markers in the subpopulation of cells surviving acute 48 -hour treatment. In both cell lines, the percentage of MYOD1+ NOG+ cells increases in a dose-dependent manner with either treatment (Figures 1(a) and 1(b)). Specifically, at the highest Vincristine treatment dose, the proportion of MYOD1+ NOG+ increased 2.4 times $(p<0.001)$ and 5.9 times $(p<0.05)$ for $\mathrm{RD}$ and A-204, respectively (Figure 1(b), top panel). Doxorubicin elicited a similar effect, with an increase of the percentage of MYOD1+ NOG+ of 4.1 times $(p<0.001)$ and 16.6 times $(p<0.01)$ in $\mathrm{RD}$ and A-204, respectively (Figure 1(b), bottom panel). Accordingly, a dose-dependent increase in Noggin mRNA levels for both cell lines was observed (Supplementary Figures S1(a) and S1(b)). Interestingly, a dose-dependent increase was observed in MYOD1 mRNA levels in the A-204 cell line (Figure S1(b)), while in $\mathrm{RD}, \mathrm{mRNA}$ levels were fluctuating resulting in not significantly different means. Currently, there are no cell membrane markers specific for MYOD1/NOG subpopulations analyzed in this study (MYOD1 is a transcription factor and Noggin is a secreted protein) preventing live cells sorting (e.g., by fluorescent activated cell sorting, FACS) for functional analysis. However, we addressed primary resistance associated with each marker by calculating the absolute cell number of each cell subpopulation by flow cytometry in the whole population treated with scalar doses of Vincristine (Supplementary Figures S2 and S3 and supplementary methods). We observed that IC50 in RD cell line for the whole population was $0.7 \mathrm{nM}$ and for MYOD1NOG- and MYOD- NOG+ subpopulations was below $0.5 \mathrm{nM}$, while there was no reduction in the cell number of MYOD1+ NOG+ subpopulation (Supplementary Figure S2). Similar response pattern was observed with A-204 (Figure S3). Doxorubicin at doses above 1uM has high levels of autofluorescence in a wide wavelength range ([34] and personal observation), thus precluding the same doseresponse analysis with this drug by flow cytometry. However, the percentages of MYOD $1+$ NOG+ cells at $0.1 \mu \mathrm{M}$ and $1 \mu \mathrm{M}$ Doxorubicin (Figure 1) increased in a dose-dependent manner. Analyzing protein level of BCL2, a well characterized antiapoptotic marker $[35,36]$, we found higher levels in the MYOD1+ NOG+ subpopulation in untreated RD and A-204 cell lines (Figure 2). In detail, we observed in both cell lines a significant 3.7 and 4.0 times higher BCL2 expression, respectively, in MYOD1+ NOG+ cells when compared to MYOD1- NOG- cells (RD: $p<0.01$; A-204: $p<0.01$ ) and 1.5 and 1.6 times when compared to MYOD1- NOG+ cells (RD: $p<0.01 ; A-204: p<0.05)$, respectively. Importantly, cells expressing only NOG and not MYOD1 also demonstrated higher BCL2 expression levels than double negative (MYOD1- NOG-) cells, suggesting the involvement of the NOG marker in chemoresistance.

3.3. TPA/GSK126 Induces Differentiation of Precursor-Like MYOD1+/NOG+ Cells and Increases Vincristine Efficacy. MYOD1+ NOG+ cells had 2.3 times increased levels of myogenin (MYOG) expression as compared to MYOD1NOG+ single positive cells $(p<0.001)$, and no MYOG was detected in MYOD- NOG- double negative cells. MYOG is 
TABle 1: Percentage of MYOD1/NOG cell subpopulations in RD and A-204 cell lines.

\begin{tabular}{lcccc}
\hline & MYOD1- NOG- & MYOD1+ NOG- & MYOD1- NOG+ & MYOD1+ NOG+ \\
\hline RD $(n=13)$ & $29.3 \pm 36.4$ & $0.5 \pm 0.9$ & $59.8 \pm 36.2$ & $10.4 \pm 13.8$ \\
A-204 $(n=13)$ & $27.2 \pm 35.6$ & $0.2 \pm 0.4$ & $69.3 \pm 35.6$ & $3.4 \pm 4.2$ \\
\hline
\end{tabular}

Cells were harvested for the analysis when cell line cultures reached $70-80 \%$ confluence. Data are presented as mean \pm standard deviation. $n$ : the number of independent observations.
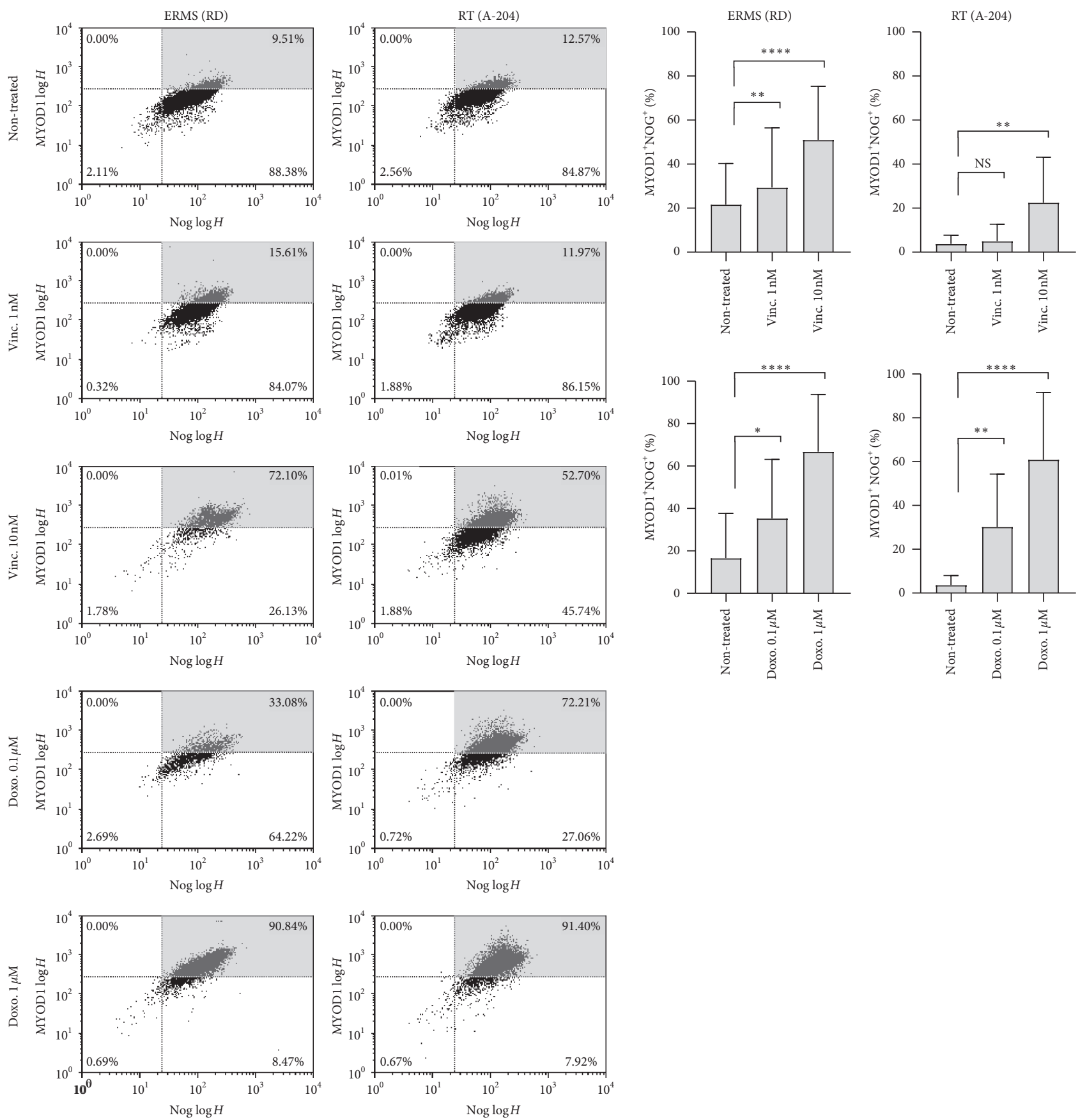

(a)

FIGURE 1: Continued.

(b) 


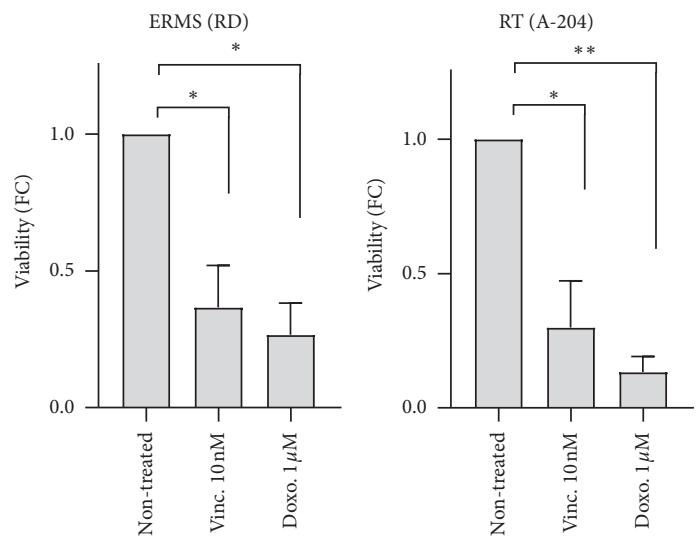

(c)

FIGURE 1: MYOD1+ NOG+ cells predominant survival in RD and A-204 cell cultures after treatment with either Vincristine or Doxorubicin. (a) Plots show examples of flow analysis of RD and A-204 cells treated with Vincristine and Doxorubicin. In grey is highlighted the quadrant representing MYOD1+ NOG+ cells. (b) Percentage of live MYOD1+ NOG+ phenotype cells in RD and A-204 cell cultures after 2 days of treatment either with 1 and $10 \mathrm{nM}$ of Vincristine (top panel) or with 0.1 and $1 \mu \mathrm{M}$ of Doxorubicin (bottom panel). (c) Absolute numbers of total live cells in RD and A-204 cultures treated with $10 \mathrm{nM}$ of Vincristine or $1 \mu \mathrm{M}$ of Doxorubicin for 2 days. Data are presented as a ratio of absolute numbers of cells in treated cultures to absolute numbers of cells in nontreated cultures (fold change). Data are shown as mean \pm standard deviation $(N=11) .{ }^{*} p<0.05,{ }^{* *} p<0.01,{ }^{* * *} p<0.001$, ANOVA with Tukey's multiple comparison test.

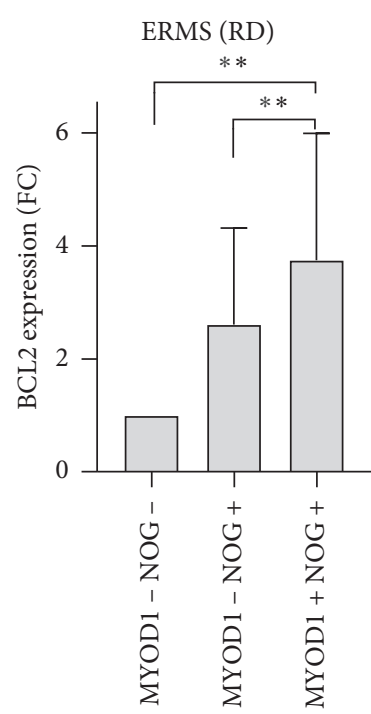

(a)

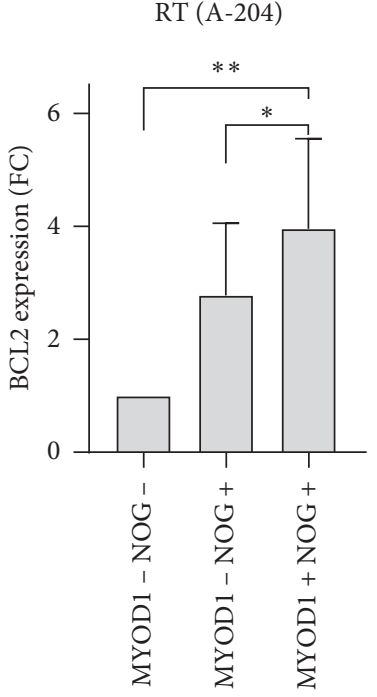

(b)

Figure 2: MYOD1+ NOG+ cells express the highest levels of antiapoptotic BCL2 protein among other cells in RD and A-204 cultures. Median fluorescence intensity (MFI) of BCL2 protein in MYOD1/NOG cell subpopulations of RD (left plot) and A-204 (right plot) cell lines. Data are presented as a ratio of BCL2 MFI of MYOD1+ NOG+ cells and MYOD1- NOG+ cells to BCL2 MFI of MYOD1- NOG- cells (fold change), (mean \pm standard deviation, $N=9$ ). ${ }^{*} p<0.05,{ }^{* *} p<0.01$, ANOVA with Tukey's multiple comparison test).

one of the myogenic regulatory factors that drive skeletal muscle cell differentiation [37, 38]. MYOG expression in cells follows and overlaps with the expression of MYOD1 in a tight temporal manner at later stages of myogenesis, and it has an indispensable role in terminal differentiation of myoblasts [39]. Importantly, MYOD1+ NOG+ cells had 2.0 times higher level of inhibitor of differentiation 1 (ID1) expression (ID1 mean fluorescence intensity) as compared to MYOD1- NOG+ single positive cells $(p<0.01)$ as assessed by flow cytometry.
Since resistant-to-chemotherapy MYOD1+ NOG+ cells demonstrated features of undifferentiated cells and their percentage increased upon chemotherapy, a combinedtreatment approach of Vincristine and 12-O-tetradecanoylphorbol-13-acetate (TPA)/GSK126 (TPA/GSK126) was tested. TPA/GSK126 has been shown to induce differentiation of RMS cell lines [31] but has not yet been tested in combination with chemotherapy. After a 6-day TPA/ GSK126 treatment, there was a statistically significant increase in the level of MYOG protein in MYOD1+ NOG+ 
cells, indicating induction of differentiation. Specifically, in RD cell line, MYOG expression increased 1.2 times in TPA/ GSK126-treated MYOD1+ NOG+ cells as compared to untreated MYOD1+ NOG+ cells $(p<0.001)$, and in A-204 cell line 1.3 times, correspondingly $(p<0.01)$. Although the average upregulation of MYOG expression was modest, at about 20\% increase, the dispersion of MYOG protein levels among cell subpopulations in $\mathrm{RD}$ was strongly reduced in MYOD1+ NOG+ cells upon treatment, indicating a robust induction of its expression in cells with low levels of MYOG (up to 2 folds). TPA/GSK126 treatment not only induced expression of MYOG suggesting cell differentiation but also exhibited a therapeutic effect by reducing on average 2 -fold absolute numbers of live cells in RMS cell cultures $(p<0.001)$ (Figure 3). Importantly, when administered in combination with Vincristine, an increased efficacy by further reduction of cell viability was observed. Specifically, in $\mathrm{RD}$ Vincristine alone decreased the number of live cells 6.7 times, as compared to nontreated cells, TPA/GSK126 2 times, and the combination of Vincristine with TPA/ GSK126 9.7 times, correspondingly (Figure 3). In RD the combination of Vincristine and TPA/GSK126 was therefore 1.5 times more effective than Vincristine alone and 4.8 times more effective than TPA/GSK126 alone. In A-204 cell cultures Vincristine decreased the number of live cells 6.2 times as compared to nontreated cells, TPA/GSK126 3.1 times, and the combination of Vincristine with TPA/ GSK126 14.2 times, a 2.3-time increase in effectiveness of Vincristine and 4.6-time increase in effectiveness of TPA/ GSK126.

\section{Discussion}

Treatment of RMS with standard chemotherapeutic agents often fails due to onset of resistance and recurrence. Since RMS tumor cells demonstrate an intratumor differential response to these agents, a degree of heterogeneity among tumor cells can be postulated. In this in vitro study, Vincristine and Doxorubicin chemoresistant subpopulations were identified as cells expressing the markers MYOD1 and NOG in two patient-derived soft-tissue tumor models (A204, a rhabdoid tumor cell line, and $\mathrm{RD}$, an embryonal rhabdomyosarcoma cell line). MYOD1+ NOG+ cell subpopulation demonstrated increased levels of BCL2 protein expression prior to treatment, thus indicating that resistance to cell death is an intrinsic property of this cell subpopulation, resulting in primary resistance to chemotherapeutic agents.

RMS and RT cells aberrantly proliferate and retain undifferentiated features. Nonetheless, within the tumor, cells may have different degrees of differentiation. The dynamic expression of MYOD1/NOG markers suggests that these cells may behave as early progenitors able to sustain tumor growth. MYOG is one of the myogenic regulatory factors that is downstream of MEF2, MYF5, MRF4, and MYOD1 that drive skeletal muscle cell differentiation $[37,38]$. MYOG expression in cells follows and overlaps with the expression of MYOD1 in a tight temporal manner at later stages of myogenesis and plays an indispensable role in

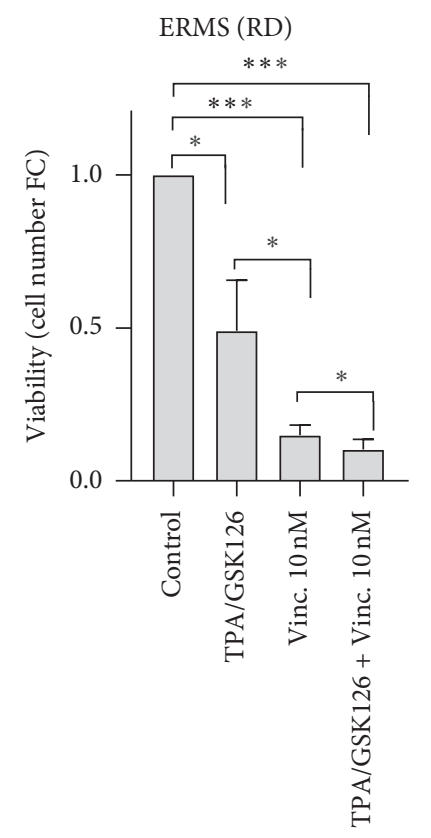

(a)

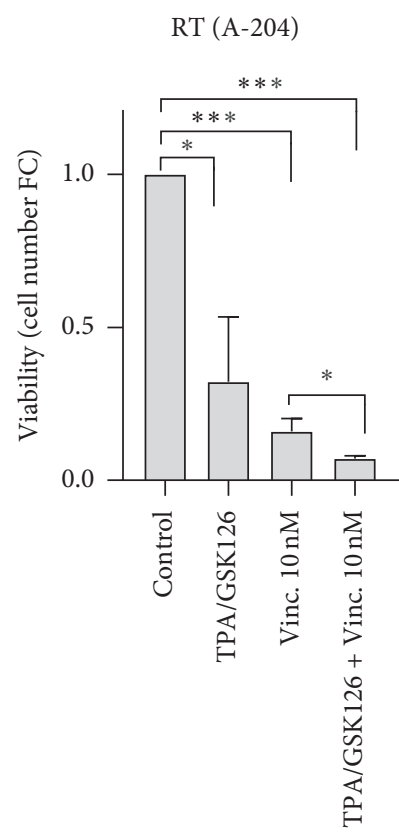

(b)
FIgURE 3: Differentiation therapy increases the effectiveness of chemotherapy. Absolute numbers of total live cells RD (a) and A204 (b) in cultures treated for 6 days with TPA/GSK126, $10 \mathrm{nM}$ of Vincristine, or combination of Vincristine and TPA/GSK126. Data are presented as a ratio of absolute numbers of cells in treated cultures to absolute numbers of cells in nontreated cultures (fold change) (mean \pm standard deviation, $N=4$ ). ${ }^{*} p<0.05$, ${ }^{* * *} p<0.001$, ANOVA with Tukey's multiple comparison test for the analysis between the groups.

terminal differentiation of myoblasts [39]. Additionally, these results demonstrated that MYOD1/NOG double positive cells are less differentiated than the rest of the cells. Since RMS is considered to be a result of failure of precursor cells to complete the differentiation process to skeletal muscle, the possibility of using differentiation therapy to address the problem of MYOD1/NOG chemoresistant cells was investigated. The concept of differentiation therapy was proposed as early as the 1970s based on the idea that the malignant cells (perhaps Cancer Stem Cells or Tumor Propagating Cells) could differentiate into less aggressive or benign cells, allowing differentiation to be an alternative or complementary strategy to chemotherapy-mediated cytotoxicity [40-42]. It has been shown that differentiation therapy can reduce malignancy by preventing tissue invasion and metastasis [43]. Leukemia is by far the most studied cancer for differentiation therapy and the only cancer with a successful application [44]. However, with solid tumors this strategy has shown limited results $[45,46]$ and it has therefore been proposed as a potential adjuvant to other therapies. A few studies have evaluated a differentiation therapy strategy in various sarcomas [47-51], but none have evaluated such approach in combination with standard chemotherapy and its effects on chemoresistant cells. Increased ID1 protein expression is characteristic of precursor cells $[25,52]$. Altogether this data suggest that 
MYOD1+ NOG+ cells are cells halted in the process of differentiation, expressing high levels of MYOG and ID1.

Since RD and A-204 MYOD1+ NOG+ cells (1) demonstrate resistance to chemotherapy and (2) show features of undifferentiated cells and (3) their percentage increases upon chemotherapy, Vincristine was tested in combination with 12O-tetradecanoylphorbol-13-acetate (TPA) and GSK126 (TPA/ GSK126). TPA/GSK126 has been shown to induce differentiation of RMS cell lines [31] but has not yet been tested in combination with chemotherapy. TPA promotes growth arrest and skeletal muscle differentiation in $\mathrm{RD}$ [53] via $\mathrm{PKC} \alpha$ activation [54]. GSK126 is a selective inhibitor of EZH2, a histone methyltransferase that epigenetically suppresses the transcription of myogenic genes [48]. Here, in addition to the direct effect of the differentiating agent TPA/GSK126, the effect of their combination with chemotherapy on a chemoresistant cell population was examined. The differentiating agent TPA/ GSK126 induced expression of the differentiation marker MYOG in RD and A-204 cells. When in combination with Vincristine, increased cell death compared to Vincristine or TPA/GSK126 alone was demonstrated.

The mechanism(s) by which expression of MYOD1 and Noggin affects chemoresistance requires further elucidation. MYOD1 and NOG might not be actively involved in the process, but could be indicators of, or associated with, other more fundamental mechanisms. The coexpression and positive correlation of MYOD1, NOG, and BCL2 expression levels warrant further mechanistic investigations.

\section{Conclusions}

In this study, the myogenic determination factor 1 (MYOD1) and the morphogenetic protein Noggin (NOG) were investigated in an embryonal rhabdomyosarcoma (ERMS) and a rhabdoid tumor (RT) cell line. Advanced, recurrent, and/or metastatic RMS and RT exhibit poor response to treatment. One of the main mechanisms behind resistance to treatment is believed to be intratumoral heterogeneity. Heterogeneous expression levels of MYOD1 and NOG were observed in both cell lines, with the MYOD1+ NOG+ subpopulation of cells crucially showing primary resistance to Vincristine and Doxorubicin, two commonly used chemotherapies for ERMS and RT. In addition to the expression of the antiapoptotic marker BCL2 indicating chemoresistance, MYOD1+ NOG+ cells expressed markers of undifferentiated cells such as ID1 and MYOG. Subsequent testing of a 3-drug combination of Vincristine with the TPA/ GSK126 differentiation therapy approach demonstrated a partial override of cells chemoresistance.

\section{Data Availability}

All flow cytometry data, cell counts, and viability data used to support the findings of this study are available from the corresponding author upon request.

\section{Conflicts of Interest}

The authors declare no conflicts of interest.

\section{Acknowledgments}

The authors thank Dr. Andrea Bottaro from Cooper Medical School of Rowan University for critical reading of the manuscript. This work was supported by Cooper Foundation.

\section{Supplementary Materials}

Figure S1: relative MYOD1 and Noggin mRNA expression levels in treated ERMS and RT cell lines. Figure S2: doseresponse curves of ERMS RD cell line to Vincristine treatment. Figure S3: dose-response curves of RT A-204 cell line to Vincristine treatment. (Supplementary Materials)

\section{References}

[1] E. Bompas, L. Campion, A. Italiano et al., "Outcome of 449 adult patients with rhabdomyosarcoma: an observational ambispective nationwide study," Cancer Medicine, vol. 7, no. 8, pp. 4023-4035, 2018.

[2] S. Ognjanovic, A. M. Linabery, B. Charbonneau, and J. A. Ross, "Trends in childhood rhabdomyosarcoma incidence and survival in the United States, 1975-2005," Cancer, vol. 115, no. 18, pp. 4218-4226, 2009.

[3] S. E. Lychou, G. G. Gustafsson, and G. E. Ljungman, "Higher rates of metastatic disease may explain the declining trend in Swedish paediatric rhabdomyosarcoma survival rates," Acta Paediatrica, vol. 105, no. 1, pp. 74-81, 2016.

[4] G. Pastore, R. Peris-Bonet, M. Carli, C. Martínez-García, J. S. de Toledo, and E. Steliarova-Foucher, "Childhood soft tissue sarcomas incidence and survival in European children (1978-1997): report from the automated childhood cancer information system project," European Journal of Cancer, vol. 42, no. 13, pp. 2136-2149, 2006.

[5] I. Sultan, I. Qaddoumi, S. Yaser, C. Rodriguez-Galindo, and A. Ferrari, "Comparing adult and pediatric rhabdomyosarcoma in the surveillance, epidemiology and end results program, 1973 to 2005: an analysis of 2,600 patients," Journal of Clinical Oncology, vol. 27, no. 20, pp. 3391-3397, 2009.

[6] Treating Rhabdomyosarcoma, American Cancer Society, 2019, https://www.cancer.org/cancer/rhabdomyosarcoma/treating. html.

[7] N. K. Gerber, L. H. Wexler, S. Singer et al., "Adult rhabdomyosarcoma survival improved with treatment on multimodality protocols," International Journal of Radiation Oncology*Biology*Physics, vol. 86, no. 1, pp. 58-63, 2013.

[8] K. Hinohara and K. Polyak, "Intratumoral heterogeneity: more than just mutations," Trends in Cell Biology, vol. 29, no. 7, pp. 569-579, 2019.

[9] X.-x. Sun and Q. Yu, "Intra-tumor heterogeneity of cancer cells and its implications for cancer treatment," Acta Pharmacologica Sinica, vol. 36, no. 10, pp. 1219-1227, 2015.

[10] B. Rybinski and K. Yun, "Addressing intra-tumoral heterogeneity and therapy resistance," Oncotarget, vol. 7, pp. 72322-72342, 2016.

[11] M. Greaves and C. C. Maley, "Clonal evolution in cancer," Nature, vol. 481, no. 7381, pp. 306-313, 2012.

[12] M. Wachtel, J. Rakic, M. Okoniewski, P. Bode, F. Niggli, and B. W. Schäfer, "FGFR4 signaling couples to Bim and not Bmf to discriminate subsets of alveolar rhabdomyosarcoma cells," International Journal of Cancer, vol. 135, no. 7, pp. 1543-1552, 2014. 
[13] A. Franco, K. N. Lewis, and J. R. Lee, "Pediatric rhabdomyosarcoma at presentation: can cross-sectional imaging findings predict pathologic tumor subtype?" European Journal of Radiology, vol. 80, no. 3, pp. e446-e450, 2011.

[14] H.-J. E. Chun, E. L. Lim, A. Heravi-Moussavi et al., "Genomewide profiles of extra-cranial malignant rhabdoid tumors reveal heterogeneity and dysregulated developmental pathways," Cancer Cell, vol. 29, no. 3, pp. 394-406, 2016.

[15] C. Walther, M. Mayrhofer, J. Nilsson et al., "Genetic heterogeneity in rhabdomyosarcoma revealed by SNP array analysis," Genes, Chromosomes and Cancer, vol. 55, no. 1, pp. 3-15, 2016.

[16] M. S. Ignatius, E. Chen, N. M. Elpek et al., "In vivo imaging of tumor-propagating cells, regional tumor heterogeneity, and dynamic cell movements in embryonal rhabdomyosarcoma," Cancer Cell, vol. 21, no. 5, pp. 680-693, 2012.

[17] Q. Tang, J. C. Moore, M. S. Ignatius et al., "Imaging tumour cell heterogeneity following cell transplantation into optically clear immune-deficient zebrafish," Nature Communications, vol. 7, p. 10358, 2016.

[18] P. Dias, D. M. Parham, D. N. Shapiro, S. J. Tapscott, and P. J. Houghton, "Monoclonal antibodies to the myogenic regulatory protein MyoD1: epitope mapping and diagnostic utility," Cancer Research, vol. 52, no. 52, pp. 6431-6439, 1992.

[19] A. Hinson, R. Jones, L. Crose, B. Belyea, F. Barr, and C. Linardic, "Human rhabdomyosarcoma cell lines for rhabdomyosarcoma research: utility and pitfalls," Frontiers in Oncology, vol. 3, 2013.

[20] P. Dias, D. M. Parham, D. N. Shapiro, B. L. Webber, and P. J. Houghton, "Myogenic regulatory protein (MyoD1) expression in childhood solid tumors: diagnostic utility in rhabdomyosarcoma," The American Journal of Pathology, vol. 137, no. 6, pp. 1283-1291, 1990.

[21] C. L. Morton and P. M. Potter, "Rhabdomyosarcoma-specific expression of the herpes simplex virus thymidine kinase gene confers sensitivity to ganciclovir," The Journal of Pharmacology and Experimental Therapeutics, vol. 286, no. 2, pp. 1066-1073, 1998.

[22] J. M. Hernández-Hernández, E. G. García-González, C. E. Brun, and M. A. Rudnicki, "The myogenic regulatory factors, determinants of muscle development, cell identity and regeneration," Seminars in Cell \& Developmental Biology, vol. 72, pp. 10-18, 2017.

[23] H. Yin, F. Price, and M. A. Rudnicki, "Satellite cells and the muscle stem cell niche," Physiological Reviews, vol. 93, no. 1, pp. 23-67, 2013.

[24] N. P. Agaram, C.-L. Chen, L. Zhang, M. P. LaQuaglia, L. Wexler, and C. R. Antonescu, "RecurrentMYOD1mutations in pediatric and adult sclerosing and spindle cell rhabdomyosarcomas: evidence for a common pathogenesis," Genes, Chromosomes and Cancer, vol. 53, no. 9, pp. 779-787, 2014.

[25] D. Costamagna, H. Mommaerts, M. Sampaolesi, and P. Tylzanowski, "Noggin inactivation affects the number and differentiation potential of muscle progenitor cells in vivo," Scientific Reports, vol. 6, no. 1, p. 31949, 2016.

[26] A. Stantzou, E. Schirwis, S. Swist et al., "BMP signaling regulates satellite cell-dependent postnatal muscle growth," Development, vol. 144, no. 15, pp. 2737-2747, 2017.

[27] J. L. Clever, Y. Sakai, R. A. Wang, and D. B. Schneider, "Inefficient skeletal muscle repair in inhibitor of differentiation knockout mice suggests a crucial role for BMP signaling during adult muscle regeneration," American Journal of Physiology-Cell Physiology, vol. 298, no. 5, pp. C1087-C1099, 2010.
[28] J. Perk, A. Iavarone, and R. Benezra, "ID family of helix-loophelix proteins in cancer," Nature Reviews Cancer, vol. 5, no. 8, pp. 603-614, 2005.

[29] Y. Ono, F. Calhabeu, J. E. Morgan, T. Katagiri, H. Amthor, and P. S. Zammit, "BMP signalling permits population expansion by preventing premature myogenic differentiation in muscle satellite cells," Cell Death \& Differentiation, vol. 18, no. 2, pp. 222-234, 2011.

[30] M. Goldstein, I. Meller, and A. Orr-Urtreger, "FGFR1 overexpression in primary rhabdomyosarcoma tumors is associated with hypomethylation of a $5^{\prime} \mathrm{CpG}$ island and abnormal expression of theAKT1, NOG, and BMP4 genes," Genes, Chromosomes and Cancer, vol. 46, no. 11, pp. 1028-1038, 2007.

[31] I. Marchesi, L. Sanna, M. Fais, F. P. Fiorentino, A. Giordano, and L. Bagella, "12-O-tetradecanoylphorbol-13-acetate and EZH2 inhibition: a novel approach for promoting myogenic differentiation in embryonal rhabdomyosarcoma cells," Journal of Cellular Physiology, vol. 233, no. 3, pp. 2360-2365, 2018.

[32] S. C. Borinstein, D. Steppan, M. Hayashi et al., "Consensus and controversies regarding the treatment of rhabdomyosarcoma," Pediatric Blood \& Cancer, vol. 65, 2018.

[33] A-204 [A204] (ATCC ${ }^{\circledR}$ HTB-82 ${ }^{\mathrm{TM}}$ ): ATCC; 2019, https:// www.atcc.org/products/all/HTB-82.aspx.

[34] S. Shah, A. Chandra, A. Kaur et al., "Fluorescence properties of doxorubicin in PBS buffer and PVA films," Journal of Photochemistry and Photobiology B: Biology, vol. 170, pp. 65-69, 2017.

[35] F. Pentimalli, S. Grelli, N. Di Daniele, G. Melino, and I. Amelio, "Cell death pathologies: targeting death pathways and the immune system for cancer therapy," Genes \& Immunity, vol. 20, no. 7, pp. 539-554, 2019.

[36] C. Touzeau, P. Maciag, M. Amiot, and P. Moreau, "Targeting Bcl-2 for the treatment of multiple myeloma," Leukemia, vol. 32, no. 9, pp. 1899-1907, 2018.

[37] M. Buckingham, "Myogenic progenitor cells and skeletal myogenesis in vertebrates," Current Opinion in Genetics \& Development, vol. 16, no. 5, pp. 525-532, 2006.

[38] H. A. Asfour, M. Z. Allouh, and R. S. Said, "Myogenic regulatory factors: the orchestrators of myogenesis after 30 years of discovery," Experimental Biology and Medicine, vol. 243, no. 2, pp. 118-128, 2018.

[39] A. Myer, E. N. Olson, and W. H. Klein, "MyoD cannot compensate for the absence of myogenin during skeletal muscle differentiation in murine embryonic stem cells," Developmental Biology, vol. 229, no. 2, pp. 340-350, 2001.

[40] G. B. Pierce, "Neoplasms, differentiations and mutations," The American Journal of Pathology, vol. 77, no. 1, pp. 103-118, 1974.

[41] G. B. Pierce and L. D. Johnson, "Differentiation and cancer," In Vitro-Journal of the Tissue Culture Association, vol. 7, p. $140,1971$.

[42] S. Kauffman, "Differentiation of malignant to benign cells," Cancer Research, vol. 31, pp. 127-134, 1971.

[43] Q. Xiong, X. Wang, L. Wang et al., "BMP-2 inhibits lung metastasis of osteosarcoma: an early investigation using an orthotopic model," Oncotargets and Therapy, vol. 11, pp. 7543-7553, 2018.

[44] H. de Thé, "Differentiation therapy revisited," Nature Reviews Cancer, vol. 18, no. 2, pp. 117-127, 2018.

[45] H. Guo, C. Liu, L. Yang et al., "Morusin inhibits glioblastoma stem cell growth in vitro and in vivo through stemness attenuation, adipocyte transdifferentiation, and apoptosis 
induction," Molecular Carcinogenesis, vol. 55, no. 1, pp. 77-89, 2016.

[46] W. P. Xu, X. Zhang, and W. F. Xie, "Differentiation therapy for solid tumors," Journal of Digestive Diseases, vol. 15, no. 4, pp. 159-165, 2014.

[47] H. Basma, S. E. Ghayad, G. Rammal et al., "The synthetic retinoid ST1926 as a novel therapeutic agent in rhabdomyosarcoma," International Journal of Cancer, vol. 138, no. 6 , pp. 1528-1537, 2016.

[48] R. Ciarapica, E. Carcarino, L. Adesso et al., "Pharmacological inhibition of EZH2 as a promising differentiation therapy in embryonal RMS," BMC Cancer, vol. 14, 2014.

[49] D. Han, V. Rodriguez-Bravo, E. Charytonowicz et al., "Targeting sarcoma tumor-initiating cells through differentiation therapy," Stem Cell Research, vol. 21, pp. 117-123, 2017.

[50] R. Taulli, F. Bersani, V. Foglizzo et al., "The muscle-specific microRNA miR-206 blocks human rhabdomyosarcoma growth in xenotransplanted mice by promoting myogenic differentiation," The Journal of Clinical Investigation, vol. 119, no. 8, pp. 2366-2378, 2009.

[51] T. Nakai, Y. Imura, H. Tamiya et al., "Trabectedin is a promising antitumor agent potentially inducing melanocytic differentiation for clear cell sarcoma," Cancer Medicine, vol. 6, no. 9, pp. 2121-2130, 2017.

[52] Y. Ono, V. F. Gnocchi, P. S. Zammit, and R. Nagatomi, "Presenilin-1 acts via Id1 to regulate the function of muscle satellite cells in a $\gamma$-secretase-independent manner," Journal of Cell Science, vol. 122, no. 24, pp. 4427-4438, 2009.

[53] S. Aguanno, M. Bouchè, S. Adamo, and M. Molinaro, "12-Otetradecanoylphorbol-13-acetate-induced differentiation of a human rhabdomyosarcoma cell line," Cancer Research, vol. 50, pp. 3377-3382, 1990.

[54] M. Bouché, F. Zappelli, M. Polimeni et al., "Rapid activation and down-regulation of protein kinase $\mathrm{C}$ alpha in 12-OTetradecanoylphorbol-13-acetate-induced differentiation of human rhabdomyosarcoma cells," Cell Growth Differentiation, vol. 6, no. 7, pp. 845-852, 1995. 